\title{
The continuation method for calculation of continuous beams of arbitrary bending rigidity
}

\author{
Andrei Verameichyk ${ }^{1}$, Mikhael Mazyrka ${ }^{2 *}$, and Vitaliy Khvisevich ${ }^{1}$ \\ ${ }^{1}$ Brest State Technical University, Moskovskaya str. 267, 224017, Brest, Belarus \\ ${ }^{2}$ PJSC «Kirovsky Zavod», Stachek Avenue, 47, 198097, St. Petersburg, Russia
}

\begin{abstract}
Abstarct. This article presents an effective mathematical continuation method for the numerical implementation of the multipoint boundary value problem, to which the calculation of a beam of arbitrary rigidity at any of its supports is reduced. The problem can be treated as a direct one in the matter of constructing an optimal design based on beam systems. A test example of the calculation is given.
\end{abstract}

\section{Introduction}

In computational practice, it is often necessary to carry out a strength analysis of structural elements whose computational scheme can be reduced to a multispan beam of variable rigidity under a variety of conditions of its support and loading $[1,2]$. Obtaining an analytical solution of such problems is associated with certain, sometimes unresolved difficulties due to the fact that the differential equation of beam bending in this case has variable coefficients and, in addition, its solutions are not continuous.

This paper presents the calculation of irregular beam systems based on the continuation method [3]. Matrix algebra allows various transformations with systems of linear algebraic and differential equations in a compact form and contributes to reducing the volume of calculations by putting them into a clear and simple form. The main advantages of the continuation method are as follows: wide variation of the type and number of supports, span lengths, and other parameters without having to repeat the calculation; calculation of complex redundant frameworks by a standard algorithm, without solving systems of symmetric equations, without reducing the calculation accuracy.

The calculation is based on the following assumptions:

- the system has any number of spans of different lengths;

- supports of any type - rigid or resilient ones;

- the end supports can be restrained, hinged immovable, hinged movable, or simple;

- the intermediate supports of the beam have horizontal mobility;

- the system is subjected to load distributed along the length, concentrated forces, moments;

- in general, the beam is of a stepped and variable cross-section;

- the system is linear and deformable, i.e. the generalized Hooke's law is valid.

*Corresponding author:mmazyrka@yandex.by 
Based on the above method, a program for the calculation of continuous beams of arbitrary bending rigidity is made, and the automated software package "ASSISTANT" [4, 5], which includes the calculation of beams, shafts, stepped beams, and other elastic systems, is created.

\section{Setting up a problem. Continuation method. Continuation for- mula}

The continuation method is based on the differential equation of the problem and its solution with subsequent derivatives.

The differential equation for the beam bending is written as [1]: $\left[E I(x) y^{\prime \prime}(x)\right]^{\prime \prime}=F(x)$, where $I(x)$ - variable moment of inertia; $E$ - modulus of elasticity, $F(x)$-specified load.

At a constant moment of inertia of the $I_{k} k$-th section of the beam and with the action of a distributed load $q$ the differential equation can be conceived of as: $E I_{k} y^{I V}(x)=q(x)$.

Application of the matrix formulation and especially obtaining on this basis the matrices of sections (spans) and transitions (supports) in combination with the use of the matrix formula of continuation form the algorithm of boundary problems solution - continuation method based on the matrix dependence

$$
\mathbf{Y}_{K}=P \cdot \mathbf{Y}_{0} \text {, }
$$

where $\mathbf{Y}_{0}$ - initial stress-strain state; $\mathbf{Y}_{K}$ - stress-strain state in the $n$-th section of the system; $P$ - impact (bending) matrix, which is the product of the corresponding span and transition matrices obtained by the continuation formula.

Let us consider the range of existence of the variable $x$ (Fig. 1), consisting of any number of intervals of length $l_{i}, i=(1,2, \ldots, \mathrm{n})$, while the end of the previous interval coincides with the beginning of the next one. Such separation is defined by internal interpolating nodes $x_{i}$, where it is necessary to determine the solution vector $\mathbf{Y}_{i}$ of the differential equation:

$$
L_{n}[\mathbf{Y}]=\mathbf{R}(x)
$$

at specified boundary conditions

$$
K_{i}[\mathbf{Y}]=\gamma_{i}
$$

where $L_{n}[\mathbf{Y}], K_{i}[\mathbf{Y}]$ - linear differential operators; $\mathbf{R}(x)$ - arbitrary vector function of a valid argument $x ; \gamma_{i}$ - numbers of constant type.

In general, the solution of equation (2) may have finite discontinuities at a number of points, but within intervals $x_{i-1}<\varepsilon<x_{i}$ the vector solution $\mathbf{Y}_{i}(\varepsilon)$ is continuous, bounded, and can be conceived of as [3]:

$$
\mathbf{Y}_{i}^{l}(\varepsilon)=A_{i}(\varepsilon) \mathbf{Y}_{i-1}^{r}
$$

where $A_{i}(\varepsilon)$ - matrix of the section $\left(x_{i-1}, \varepsilon\right), \mathbf{Y}_{i-1}^{r}$ - value of the vector $\mathbf{Y}_{i-1}$ to the right of the point $x_{i-1}$.

If at the point $x_{i-1}$ the solution $\mathbf{Y}_{i-1}$ has discontinuity of the first kind, then it can be written as follows:

$$
\mathbf{Y}_{i-1}^{r}=F_{i-1} \mathbf{Y}_{i-1}^{l},
$$


where $F_{i-1}-x_{i-1}$ point transition (jump) matrix, where the solution has discontinuity, $\mathbf{Y}_{i-1}^{l}$ - value of the vector $\mathbf{Y}_{i-1}$ to the left of the point $x_{i-1}$.

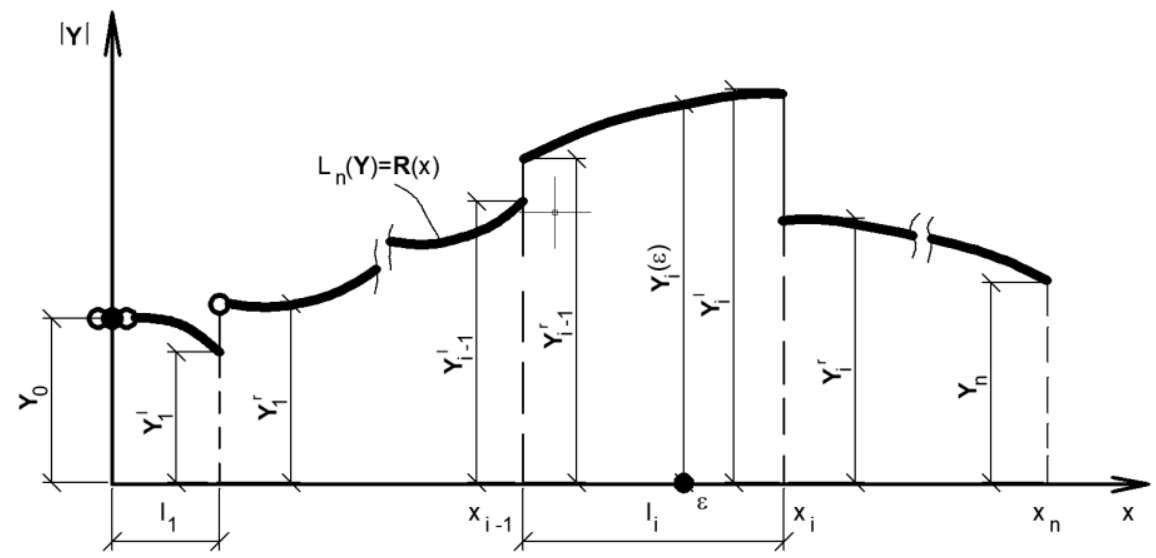

Fig. 1. To the use of the continuation method

Substituting dependence (5) into formula (4), we find the solution of equation (2) at the end of the interval (to the left of the point $x_{i}$ ), expressed in terms of the solution at the starting point $x_{i-1}$ of the considered interval:

$$
\mathbf{Y}_{i}^{l}\left(x_{i}\right)=A_{i}\left(l_{i}\right) F_{i-1} \mathbf{Y}_{i-1}^{l}\left(x_{i-1}\right) .
$$

The solution $\mathbf{Y}_{i-1}^{l}$ included in this formula can also be conceived of as:

$$
\mathbf{Y}_{i-1}^{l}\left(x_{i}\right)=A_{i-1}\left(l_{i-1}\right) F_{i-2} \mathbf{Y}_{i-2}^{l}\left(x_{i-2}\right) \text {. }
$$

Based on formulas (6) and (7) for the boundary value problem (2), the boundary conditions $\mathbf{Y}_{n}$ and $\mathbf{Y}_{0}$ can be related by the dependence:

$$
\mathbf{Y}_{n}^{l}\left(x_{n}\right)=A_{n}\left(l_{n}\right) F_{n-1} A_{n-1}\left(l_{n-1}\right) F_{n-2} \ldots A_{2}\left(l_{2}\right) F_{1} A_{1}\left(l_{1}\right) F_{0} \mathbf{Y}_{0}^{l},
$$

or

$$
\mathbf{Y}_{n}^{l}\left(x_{n}\right)=\prod_{i=n}^{1}\left[A_{i}\left(l_{i}\right) F_{i-1}\right] \mathbf{Y}_{0}^{l} .
$$

The relation (8) expresses the mathematical meaning of the continuation method and is called the continuation formula. The $A_{i}\left(l_{i}\right)$ section matrices included here are determined from differential equation (2), written for each specific $i$-th section. The jump matrices are determined from the matching conditions of the limiting points of adjacent intervals and become singular ones at $i \neq j$. Consequently, to obtain the desired solution $\mathbf{Y}_{i}=\mathbf{Y}_{i}(x)$, it is necessary to calculate the product of a chain of matrices $A_{i}\left(l_{i}\right) F_{i-1}$ and satisfy the boundary conditions.

\section{Matrices of sections, jumps, and transition through intermedi- ate supports}

The $A_{i}\left(l_{i}\right)$ section matrices included in formula (8), as mentioned above, are determined from differential equation (2) written for each specific $i$-thsection. They have the following form: 


$$
A_{i}\left(l_{i}\right)=\left[\begin{array}{ccccc}
1 & l_{i} & \frac{l_{i}^{2}}{2 ! E I_{i}} & \frac{l_{i}^{3}}{3 ! E I_{i}} & \frac{q_{i} l_{i}^{4}}{4 ! E I_{i}} \\
0 & 1 & \frac{l_{i}}{E I_{i}} & \frac{l_{i}^{2}}{2 ! E I_{i}} & \frac{q_{i} l_{i}^{3}}{3 ! E I_{i}} \\
0 & 0 & 1 & l_{i} & \frac{q_{i} l_{i}^{2}}{2 !} \\
0 & 0 & 0 & 1 & q_{i} l_{i} \\
0 & 0 & 0 & 0 & 1
\end{array}\right] .
$$

The $F_{i}$ jump matrices are determined by considering the matching conditions of the limiting points of adjacent intervals. Since the partition of the beam into sections is carried out in such a way that all the external concentrated forces and moments fall into the $j$-th node points (limiting points of the sections), the static components of the $\mathbf{Y}_{i}$ vectors undergo discontinuities when passing through these points, whereas:

$$
\mathbf{Y}_{i}^{r}=F_{j} \mathbf{Y}_{i}^{l} .
$$

Where $F_{j}-$ transition (jump) matrix:

$$
F_{j}=\left[\begin{array}{ccccc}
1 & 0 & 0 & 0 & 0 \\
0 & 1 & 0 & 0 & 0 \\
0 & 0 & 1 & 0 & M_{j} \\
0 & 0 & 0 & 1 & P_{j} \\
0 & 0 & 0 & 0 & 1
\end{array}\right],
$$

where $M_{j}, P_{j}$ - external concentrated moments and forces.

The stress-strain state vector at an arbitrary point $j$ of the beam is written as:

$$
\left[\mathbf{Y}_{j}\right]=\left[\begin{array}{lllll}
W_{j} & \varphi_{j} & M_{j} & Q_{j} & 1
\end{array}\right],
$$

where $W_{j}$-deflection at the point $j, \varphi_{j}$ - rotation angle at the point $j, M_{j}, P_{j}$ - bending moment and shear force at the point $j$ respectively.

When solving boundary problems for beam systems, only two $\mathbf{Y}_{0}^{r}$ vector components can be specified. The other two components of the $\mathbf{Y}_{0}^{r}$ vector are unknown. As indeterminates we assume:

a) $M_{0}=X_{1}, Q_{0}=X_{2} \quad$ (rigidly fixing);

b) $W_{0}=X_{1}, \varphi_{0}=X_{2} \quad$ (free end);

c) $\varphi_{0}=X_{1}, Q_{0}=X_{2} \quad$ (hinged edge).

Let us represent one of the indeterminates as a linear dependence

$$
X_{1}=m_{1} X_{2}+n_{1},
$$

where $m_{1}, n_{1}$ - currently unknown coefficients.

Then any column vector of the initial state for the case when the left support is a rigid restraint can be conceived of as: 


$$
\mathbf{Y}_{0}^{r}=\left[\begin{array}{c}
0 \\
0 \\
M_{0} \\
Q_{0} \\
1
\end{array}\right]=\left[\begin{array}{c}
0 \\
0 \\
X_{1} \\
X_{2} \\
1
\end{array}\right]=\left[\begin{array}{c}
0 \\
0 \\
m_{1} X_{2}+n_{1} \\
X_{2} \\
1
\end{array}\right]=\left[\left[\begin{array}{l}
0 \\
0 \\
1 \\
0 \\
0
\end{array}\right] m_{1}+\left[\begin{array}{l}
0 \\
0 \\
0 \\
1 \\
0
\end{array}\right]\right] X_{2}+\left[\left[\begin{array}{l}
0 \\
0 \\
1 \\
0 \\
0
\end{array}\right] n_{1}+\left[\begin{array}{l}
0 \\
0 \\
0 \\
0 \\
1
\end{array}\right]\right] .
$$

In short for any case:

$$
\mathbf{Y}_{0}^{r}=\left(\mathbf{B}_{1}^{0} m_{1}+\mathbf{B}_{2}^{0}\right) X_{2}+\left(\mathbf{B}_{3}^{0} n_{1}+\mathbf{B}_{4}^{0}\right) .
$$

Vectors $\mathbf{B}_{1}^{0}, \mathbf{B}_{2}^{0}, \mathbf{B}_{3}^{0}$ and $\mathbf{B}_{4}^{0}$ are the columns of the $\mathbf{B}_{0}$ initial state matrix, have dimensions of $(3 \times 4)$. Let us determine the value of the stress-strain state vector at the first point. In this case we can write

$$
\mathbf{Y}_{1}^{l}\left(l_{1}\right)=A_{1}\left(l_{1}\right) \mathbf{Y}_{0}^{r} \text {. }
$$

Applying (14) to (15), we obtain:

$$
\begin{gathered}
\mathbf{Y}_{1}^{l}\left(l_{1}\right)=A_{1}\left(l_{1}\right)\left[\left(\mathbf{B}_{1}^{0} m_{1}+\mathbf{B}_{2}^{0}\right) X_{2}+\left(\mathbf{B}_{3}^{0} n_{1}+\mathbf{B}_{4}^{0}\right)\right]= \\
=\left[m_{1}\left(A_{1} \mathbf{B}_{1}^{0}+A_{1} \mathbf{B}_{2}^{0}\right) X_{2}\right]+\left[n_{1}\left(A_{1} \mathbf{B}_{31}^{0}\right)+A_{1} \mathbf{B}_{4}^{0}\right]= \\
=\left(m_{1} \mathbf{B}_{1}^{1}+\mathbf{B}_{2}^{1}\right) X_{2}+\left(n_{1} \mathbf{B}_{3}^{1}+\mathbf{B}_{4}^{1}\right),
\end{gathered}
$$

where $A_{1} \mathbf{B}_{p}^{0}=\mathbf{B}_{p}^{1}, p=(1,2,3,4)$.

Summarizing the result, we can write

$$
A_{i} \mathbf{B}_{p}^{i-1}=\mathbf{B}_{p}^{i} .
$$

Then, using the continuation formula (8), we can determine the matrices for all nodal points of the beam. The linear approximation coefficients $m_{1}$ and $n_{1}$ are determined at the first intermediate rigid support, where the deflection of the beam is zero, i.e.

$$
W_{j}=\left(m_{1} b_{11}^{j}+b_{12}^{j}\right) X_{2}+\left(n_{1} b_{13}^{j}+b_{14}^{j}\right)=0 .
$$

It follows therefrom that:

$$
\begin{array}{ll}
m_{1} b_{11}^{j}+b_{12}^{j}=0, & m_{1}=-\frac{b_{12}^{j}}{b_{11}^{j}}, \quad(\mathrm{j}=1) . \\
n_{1} b_{13}^{j}+b_{14}^{j}=0, & m_{1}=-\frac{b_{13}^{j}}{b_{14}^{j}},
\end{array}
$$

When passing through the first rigid support, there will be a jump in the magnitude of the shear force, numerically equal to the reaction $S_{1}$ arising in this support. Let us also represent the reaction $S_{1}$ as a linear dependence on $X_{2}$ :

$$
S_{1}=m_{2} X_{2}+n_{2}
$$

where $m_{2}$ and $n_{2}$ - currently unknown coefficients.

Then directly to the right of the first support we obtain:

$$
\begin{aligned}
\mathbf{Y}_{j}^{r} & =\left(m_{1} \mathbf{B}_{1}^{j}+\mathbf{B}_{2}^{j}\right) X_{2}+\left(n_{1} \mathbf{B}_{3}^{j}+\mathbf{B}_{4}^{j}\right)+\left[\begin{array}{c}
0 \\
0 \\
0 \\
1 \\
0
\end{array}\right] m_{2} X_{2}+\left[\begin{array}{c}
0 \\
0 \\
0 \\
1 \\
0
\end{array}\right] n_{2}= \\
& =\left(m_{1} \mathbf{B}_{1}^{j}+\mathbf{B}_{2}^{j}+m_{2} \mathbf{G}^{j}\right) X_{2}+\left(n_{1} \mathbf{B}_{3}^{j}+\mathbf{B}_{4}^{j}+n_{2} \mathbf{R}^{j}\right) .
\end{aligned}
$$


Let us introduce the designations

$$
\begin{aligned}
& \left(\mathbf{B}_{1}^{j}\right)^{r}=\mathbf{G}^{j}, \quad\left(\mathbf{B}_{2}^{j}\right)^{r}=m_{1} \mathbf{B}_{1}^{j}+\mathbf{B}_{2}^{j}, \\
& \left(\mathbf{B}_{3}^{j}\right)^{r}=\mathbf{R}^{j}, \quad\left(\mathbf{B}_{4}^{j}\right)^{r}=n_{1} \mathbf{B}_{3}^{j}+\mathbf{B}_{4}^{j} .
\end{aligned}
$$

The vectors $\left(\mathbf{B}_{1}^{j}\right)^{r},\left(\mathbf{B}_{2}^{j}\right)^{r},\left(\mathbf{B}_{3}^{j}\right)^{r}$ and $\left(\mathbf{B}_{4}^{j}\right)^{r}$ are the columns of the matrix of transition $\left(\mathbf{B}^{j}\right)^{r}$ through the rigid support.

The coefficients $m_{2}$ and $n_{2}$ are determined from the condition that the deflection at the second intermediate support $j=2$ is zero, similarly to (19). Thus, in general, the effect of any rigid intermediate support is expressed in the appearance of new unknown coefficients $m_{j}$ and $n_{j}$, determined on subsequent rigid supports $j+1$, as well as in the transformation of the matrix $B_{j}$ at the transition through the support.

At the right end of the beam in the case of rigid support and hinged edge, the coefficients $m$ and $n$ for the next to last support are determined from the condition $W_{n}=0$. In the case of a free edge, the coefficients $m$ and $n$ are determined from the condition that the bending moment is zero.

The unknown parameter $X_{2}$ is determined from the second condition at the right end of the beam. Thus, in the case of a rigid restraint at the right end of the beam, this condition is the condition that the angle of rotation $\varphi$ is zero, i.e. $\varphi_{n}=0$.

Knowing $X_{2}$, by formula (13) we find the parameter $X_{1}$, i.e. we determine completely the column vector of the initial state $\mathbf{Y}_{0}^{r}$. Using formulas similar to (20), all the unknown reactions $S_{j}$ of the intermediate supports are determined. Further calculation of a multispan beam is carried out according to the continuation formula with known reactions $S_{j}$ as external concentrated forces, i.e. as a statically determined beam.

\section{Calculation algorithm for multispan beams and its implemen- tation}

Based on the continuation method, an algorithm for calculating multispan beams of variable rigidity was compiled and a computer program was developed.

For the calculation it is necessary to break the beam into sections, within which the rigidity $E I_{i}$ and the transverse distributed load $q$ are approximately constant. Whereas, the node points coincide with the points of application of external concentrated loads and the points of installation of intermediate supports. Then the values of lengths and rigidities for each section, as well as the values of all force factors and identificators of the supports are set.

The result of the calculation of a multispan beam is certain constraint reactions (forces and concentrated moments), as well as the curves of deflections, rotation angles, bending moments and transverse forces.

A number of test problems were solved, confirming the validity of the developed algorithm. Fig. 2 shows an example of calculation of a beam, the analytical solution for which was considered in $[2, \S 117]$ with the following numerical data: $l_{1}=l_{2}=l_{3}=1 \mathrm{~m}, P=100$ $\mathrm{kN}, q=100 \mathrm{kN} / \mathrm{m}$. 


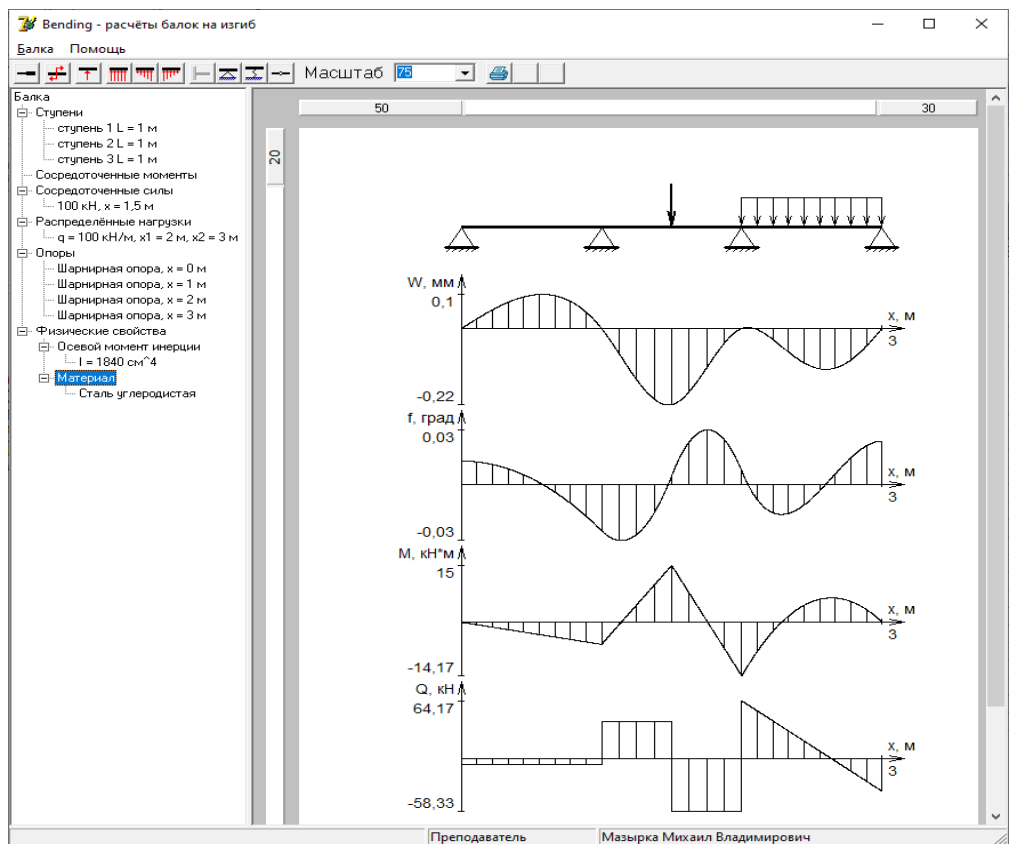

Fig. 2. An example of calculating a multiplet beam by continuing

The method considered in this paper is expected to be used in the future to optimize multispan beams of variable rigidity.

A group of authors has created an automated software package "ASSISTANT" [5] designed to automate the work of a professor involved in strength of materials and electrical engineering teaching (Fig. 3).

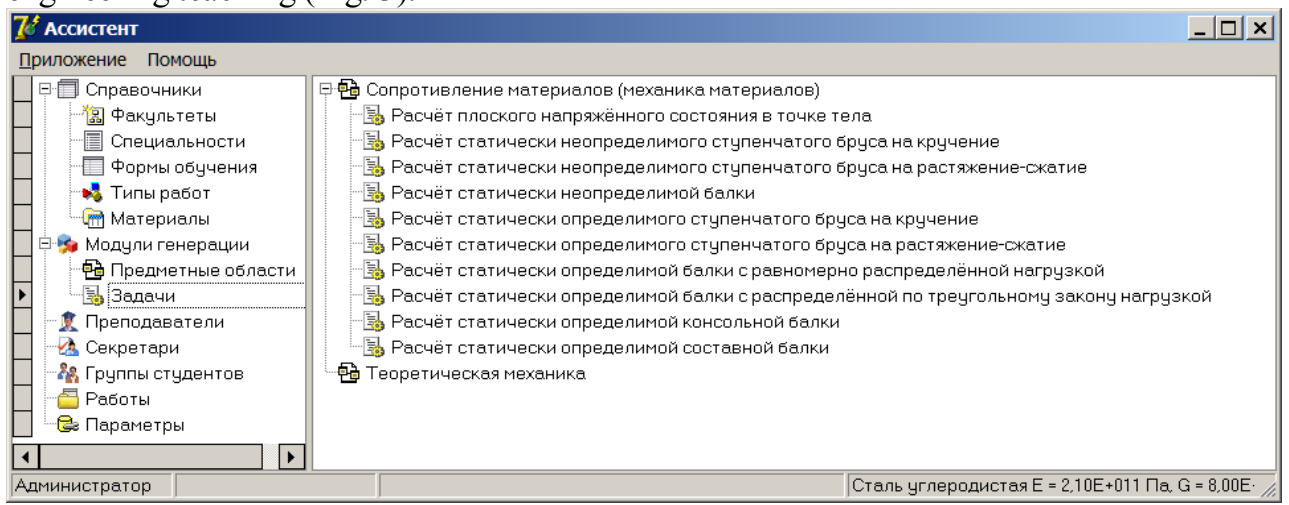

Fig. 3. Interface of the automated software package "ASSISTANT"

This package includes modules for beam bending analysis and beam tensioncompression, torsion analysis, which are based on the calculation method of continuation. It allows you to generate tasks and monitor their completion by students. The "ASSISTANT" software package can generate tasks for the following problems: calculation of statically determinate and statically indeterminate beams; calculation of statically determinate and statically indeterminate discontinuous beam in tension-compression; calculation of statically indeterminate rod system; calculation of shafts in torsion; calculation of geometric characteristics of plane section; calculation of plane stress condition in body point; calculation 
of direct and alternating current circuits, three-phase circuit, etc. The package is a multiuser system.

The use of such a system of automated teaching allows you to improve the quality of students' study of disciplines and improve student performance, as well as significantly facilitate the work of the professor and reduce the time spent on control.

\section{Conclusion}

The suggested continuation method is high accurately. Due to its versatility, the developed algorithm of the continuation method greatly simplifies and standardizes the process of analyzing complex systems with a high degree of static indeterminacy, and also allows a wide range of initial parameters of the basic system of the supporting structure in the process of calculations. The continuation method can be widely used for other elastic systems: shafts, space systems, frames, plates, shells, etc. for the main types of force action: bending, stability, vibrations, transverse-longitudinal bending.

\section{References}

1. S.P. Timoshenko Soprotivlenie materialov. Fizmatgiz (1960)

2. N.M. Belyaev Soprotivlenie materialov. Nauka (1976)

3. K.K. Ponomaryov Raschyot elementov konstrukcij s primeneniem ECVM. - M.: Mashinostroenie, 1972. - $424 \mathrm{~s}$

4. A.I. Verameichyk, M.V. Mazyrka, V.M. Hvisevich, Organizaciya samostoya-tel'noj raboty studentov s ispol'zovaniem avtomatizirovannogo programm-nogo kompleksa sb. materialov VII mezhdunar. nauch.-prakt. konf. «Vychislitel'nye metody, modeli i obrazovatel'nye tekhno-logii», Brest, 18 okt. 2019 g. (2019)

5. V.M. Hvisevich, A.I. Verameichyk, M.V. Mazyrka, Teoreticheskaya i prikladnaya mekhanika, 33 (2018) 